\title{
Endoscopic ultrasound and pancreatic applications: what the radiologist needs to know
}

\author{
Siva P. Raman, ${ }^{1}$ Elliot K. Fishman, ${ }^{1}$ Anne Marie Lennon ${ }^{2}$ \\ ${ }^{1}$ Department of Radiology, Johns Hopkins University, JHOC 3251, 601 N. Caroline Street, Baltimore, MD 21287, USA \\ ${ }^{2}$ Department of Gastroenterology, Johns Hopkins University, Sheikh Zayed Tower, Suite 7125j, Baltimore, MD 21287, USA
}

\begin{abstract}
As the technology has improved, endoscopic ultrasound (EUS) has taken on an important role in the diagnosis of a number of different neoplastic and non-neoplastic pancreatic diseases. EUS can provide high-resolution images with subtle anatomic detail, and has also taken on an important role in the targeted biopsy of the pancreas and adjacent structures. This review seeks to familiarize radiologists with the role of EUS in the diagnosis of chronic and autoimmune pancreatitis, solid pancreatic masses, and cystic pancreatic neoplasms.
\end{abstract}

Key words: Endoscopic ultrasound-Computed tomography-Pancreas-Pancreatic cancer-Pancreatitis-Neuroendocrine tumor

Radiologists are well aware of the dramatic impact multidetector computed tomography (MDCT) and magnetic resonance imaging (MRI) have had upon the diagnosis (and ultimate treatment) of pancreatic disorders. These two modalities have become firmly entrenched in the diagnostic algorithm for a large number of different pancreatic diseases, and have a major role to play in screening, diagnosis, and followup. However, most radiologists have little familiarity with the increasingly important role endoscopic ultrasound (EUS) now plays in the diagnosis and treatment of pancreatic diseases. As a result of the endoscopic probe being positioned in close proximity to the pancreas and extrahepatic biliary system, this technique allows extraordinarily high-resolution images with subtle anatomic detail, providing a level of analysis not routinely possible with either CT or MRI. Moreover,

Correspondence to: Siva P. Raman; email: srsraman3@gmail.com the technique has become a mainstay in terms of its ability to allow targeted biopsies of the pancreas and adjacent structures [1].

This review article aims to familiarize the radiology readership with the basics of EUS technique, the complementary role of EUS (to CT and MRI) in the diagnostic algorithm for a number of different pancreatic disorders and lesions, correlation between the MDCT and EUS findings for each of these disorders, and the role of EUS-guided biopsy in pancreaticobiliary diseases.

\section{Technique}

EUS is performed through the attachment of a highfrequency transducer to the tip of an endoscope, with the subsequent acquisition of transluminal images. Unlike standard transcutaneous ultrasound, where ultrasound gel is used as a coupling medium, this is not possible during the acquisition of transluminal images. Instead, a small balloon filled with water is attached to the transducer tip, and this water serves as the primary coupling medium (Fig. 1). In addition, the image quality can be further improved by the removal of air, and instillation of water, into the gut lumen $[2,3]$.

While a number of different endoscopes are available commercially, these can be divided into two broad categories: (1) Radial echoendoscopes provide the operator with a $360^{\circ}$ view perpendicular to the long axis of the endoscope, while (2) Linear array echoendoscopes provide images parallel to the long axis of the endoscope (Fig. 1). In particular, the linear array endoscope is thought to be technically more difficult for the endoscopist to maneuver, but is of great value during EUSguided FNA, allowing the visualization of the needle along its entire length $[2,3]$. High frequency probes can also be used which are inserted through the biopsy channel of a regular endoscope. These have the advantage of providing very high frequency images, and are 

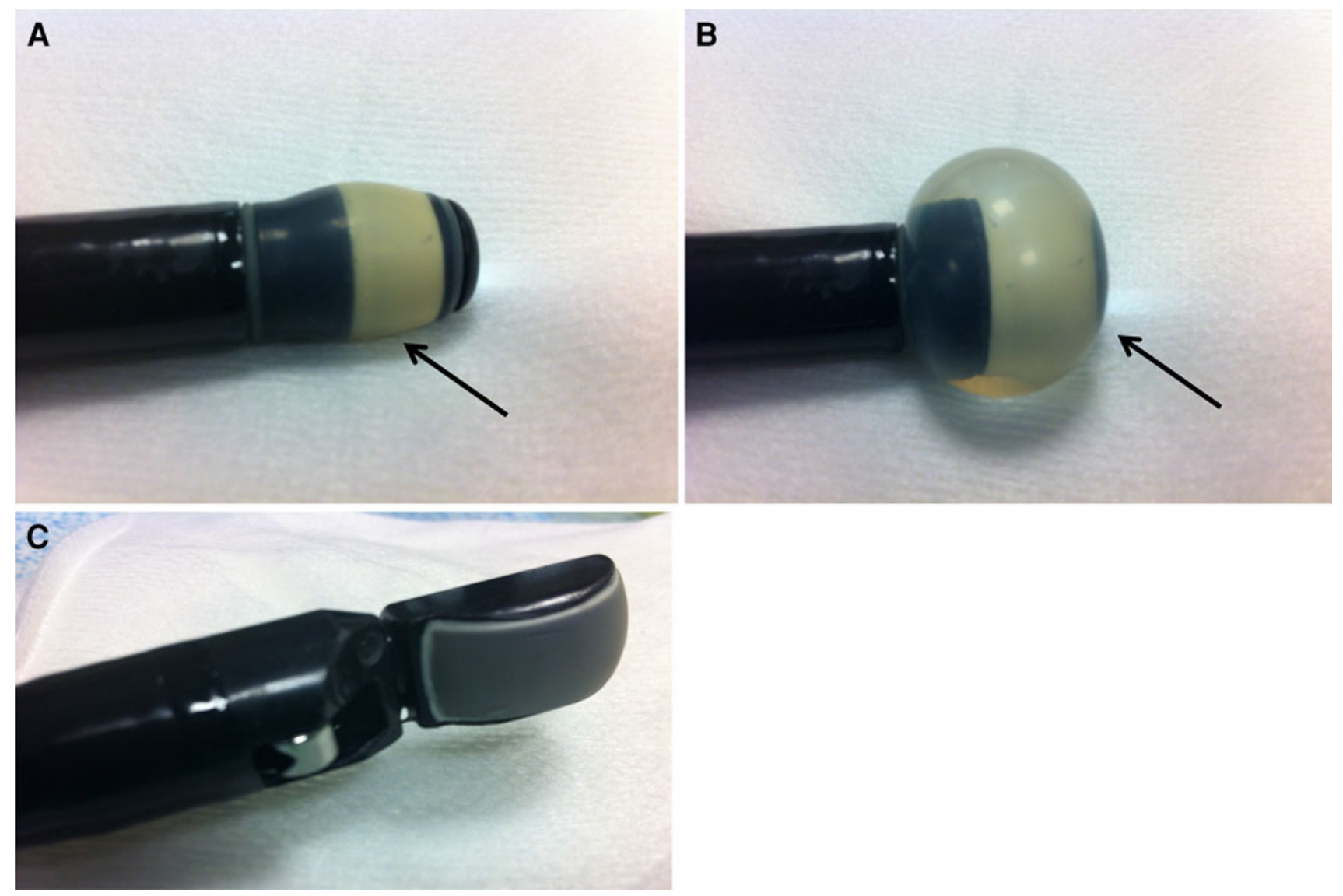

Fig. 1. Examples of endoscopic ultrasound echoendoscopes. A radial probe with the balloon (arrows) deflated (A) and inflated (B). C demonstrates a typical linear ultrasound probe.

excellent for staging early tumors, or passing through a stricture. However, due to their high frequency, these probes lack the depth penetration provided by radial or linear array echoendoscopes and can only be used to assess mucosal lesions.

EUS can be used to examine the entirety of the pancreas from the uncinate process to the tail in almost all patients, except those with an impassable duodenal stenosis or non-amenable post-surgical anatomy (such as a Billroth II anastomosis). Once the endoscope has been maneuvered into place by the endoscopist, two different sets of images are typically acquired: (1) Transgastric images are acquired with the endoscope in the gastric antrum and body, and allow imaging of the pancreatic body and tail (Figs. 2, 3, 4). As with standard transcutaneous ultrasound, the pancreas is typically hyperechoic to the liver and has a grainy echotexture. The portal vein and splenic vein are crucial landmarks to provide orientation for the endoscopist. The pancreatic duct can be seen with exquisite detail in this view running along the length of the pancreatic body and tail. The endoscopist can evaluate different portions of the pancreatic body and tail by moving the endoscope through various parts of the stomach [4]. (2) Transduodenal images are vital for evaluating the uncinate process, pancreatic head and ampulla,

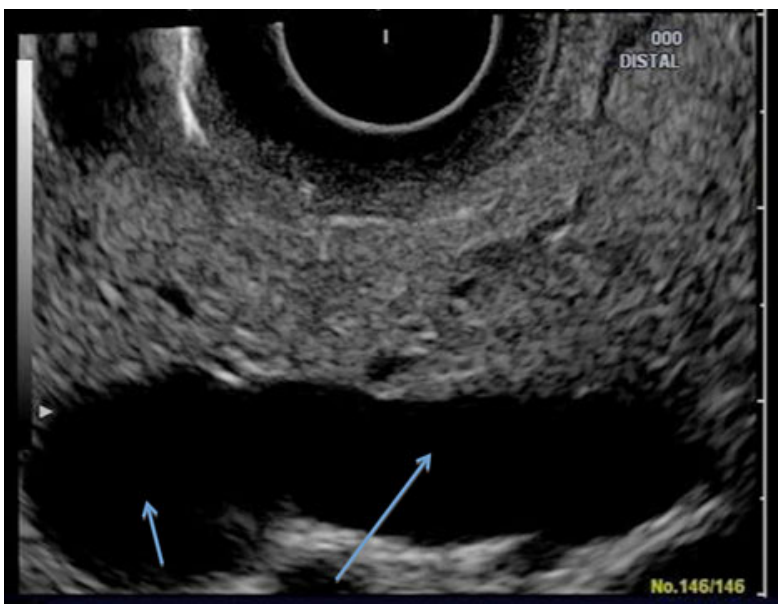

Fig. 2. Normal endoscopic ultrasound image of the pancreas acquired with a radial probe during transgastric imaging. The confluence of the splenic vein and portal vein has been termed the "club head" because its shape is similar to a golf club (short arrow-portal-splenic confluence; long arrow-splenic vein).

as well as the extrahepatic bile duct (Fig. 5). The goal in this portion of the examination is to visualize the common bile duct and pancreatic duct entering the ampulla, a view 


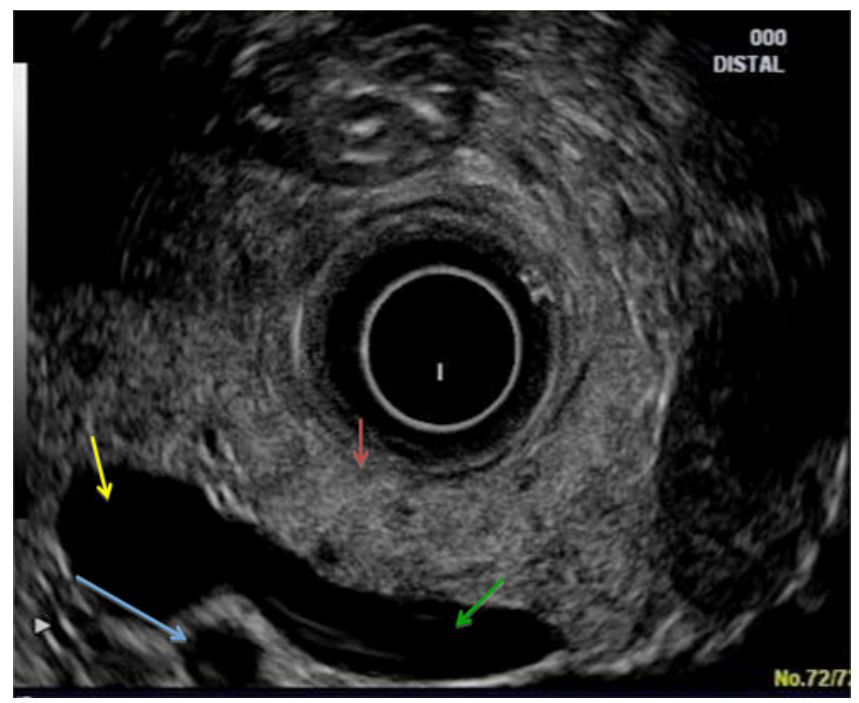

Fig. 3. Normal endoscopic ultrasound image acquired with a radial probe during trangastric imaging of the pancreatic body. The splenic vein (green arrow), confluence (yellow arrow), superior mesenteric artery (blue arrow), and pancreatic body (red arrow) are visualized.

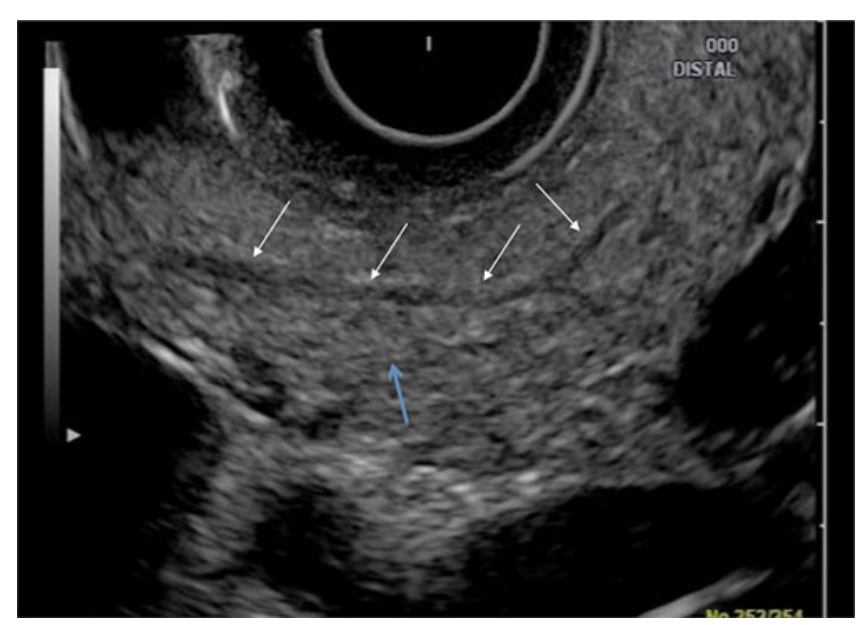

Fig. 4. Normal endoscopic ultrasound image of the pancreatic body acquired with a radial probe during transgastric imaging (blue arrow-pancreatic parenchyma; white arrows-pancreatic duct). This image illustrates the normal echotexture of the pancreas, which is mildly coarse and typically mildly hyperechoic relative to the liver.

typically accomplished by maneuvering the endoscope into the second portion of the duodenum $[2,3]$.

\section{Non-neoplastic disorders of the pancreas}

\section{Chronic pancreatitis}

In advanced cases, CT can demonstrate several characteristic findings of chronic pancreatitis, including parenchymal atrophy, pancreatic ductal dilatation with beading and irregularity, and pancreatic ductal and parenchymal calcifications. However, it is well understood that CT is not sensitive for identifying chronic pancreatitis in the earliest stages of the disease. In the earlier stages of the disorder, ERCP has traditionally been considered as the gold standard test. However, ERCP is invasive, with a risk of post ERCP pancreatitis of approximately $5 \%$, and a reported mortality rate of $0.2-0.8 \%[5,6]$. In addition, ERCP is limited to evaluation of the pancreatic duct itself, is often limited in its ability to visualize changes in pancreatic side branches, and cannot provide information regarding changes in the pancreatic parenchyma [7, 8]. By comparison, EUS is relatively non-invasive and allows assessment of the pancreatic ductal system and parenchyma.

Several different EUS features of chronic pancreatitis have been described in the pancreatic parenchyma, including parenchymal heterogeneity, prominent echogenic septations extending through the pancreas, lobulation of the pancreatic gland contour, hypoechoic or anechoic spaces in the substance of the pancreas, and bright intrapancreatic echoes. Similar to findings on CT, evaluation of the pancreatic duct often reveals dilatation of the duct $>3 \mathrm{~mm}$, ductal tortuosity and beading, increased echogenicity of the pancreatic duct walls, echogenic foci/calculi within the duct, and side branch ectasia (Fig. 6) [7]. Some of these findings can be subtle, and there can be interobserver variability in the appreciation of several of these findings (particularly the parenchymal findings) [9]. There are a small number of studies directly comparing EUS with surgical pathology. In one study of 71 patients, three or more EUS criteria had a sensitivity of $83 \%$ and a specificity of $80 \%$ for chronic pancreatitis, and the specificity increased to $100 \%$ when five or more criteria were used [10].

Depending on the number of imaging criteria utilized in the diagnosis, EUS has proven to be significantly more sensitive than CT and ERCP in the diagnosis of early cases of chronic pancreatitis. In a study by Kahl et al., 38 patients with clinically suspected chronic pancreatitis, but with a negative ERCP, were found to have evidence of chronic pancreatitis on EUS. 22 of these 32 patients were found to have evidence of chronic pancreatitis on a follow-up ERCP performed between 6 and 25 months after the initial evaluation [11]. Catalano et al. studied 37 patients with EUS evidence of chronic pancreatitis on EUS, but with normal CT studies and secretin function tests. $2 / 3$ of these patients ultimately developed evidence of chronic pancreatitis on either a follow-up CT or secretin function test $[7,12]$.

\section{Autoimmune pancreatitis}

Autoimmune pancreatitis (AIP) can be very difficult to differentiate from a neoplasm or conventional edematous pancreatitis on any imaging modality. On EUS, AIP can present as a diffusely enlarged, sausage-shaped gland 

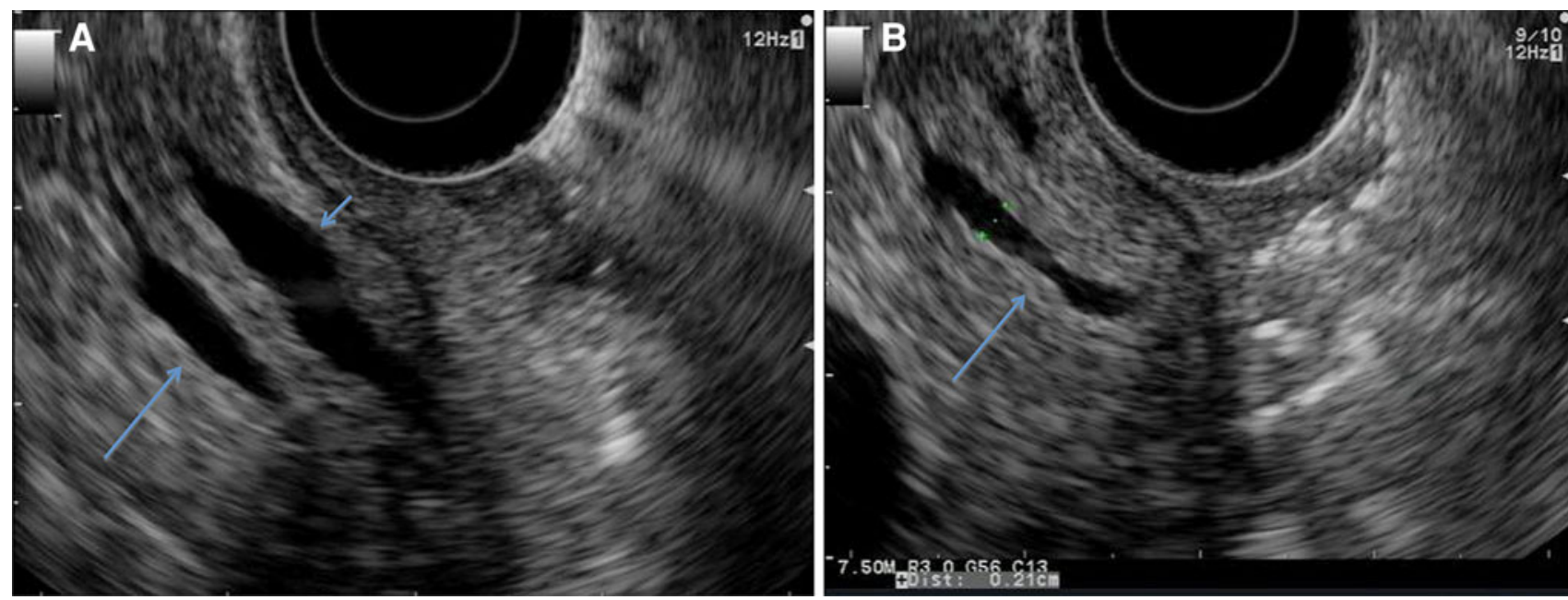

Fig. 5. A Normal endoscopic ultrasound image of the pancreatic head acquired using a radial probe during transduodenal imaging. This image demonstrates the common bile duct (short arrow) and pancreatic duct (long arrow) converging near

with hypoechoic margins, or as a focal hypoechoic mass (typically without upstream pancreatic ductal dilation) $[13,14]$. Bile duct wall thickening and strictures are common features, and are often best appreciated on EUS. Biopsies of the pancreas can be obtained using EUS, which can then be stained for IgG4, allowing a specific diagnosis [15].

\section{Solid pancreatic masses}

\section{Pancreatic neuroendocrine tumors}

EUS has an important role to play in the identification of pancreatic neuroendocrine tumors (NETs): even with good dual phase technique (arterial and venous phase images), the sensitivity of CT is only $64-82 \%$, and CT struggles in the identification of small lesions [16]. However, EUS has proven to be very sensitive in the identification of NETs, even when confronted with very small lesions measuring $5 \mathrm{~mm}$ or less. A study conducted in 1992 by Rosch et al. showed EUS to have an overall sensitivity of $82 \%$ and a specificity of $92 \%$ for NETs, while a more recent study in 2000 by Zimmer et al. demonstrated a sensitivity of $93 \%$ and a specificity of 95\% [17-19]. In other words, EUS is a valuable tool to identify NETs in those cases with a high clinical index of suspicion, but a negative CT examination [16]. More recently, EUS has also proven extraordinarily valuable in marking the site of small lesions, such as NETs, with a "tattoo" placed under EUS guidance, aiding in the identification of the lesion during laparoscopic surgery (Fig. 7) [20].

Pancreatic NETs are typically hypoechoic to the surrounding pancreatic parenchyma, although rare cases can be isoechoic, making identification more dif- the ampulla. B Normal endoscopic ultrasound image (radial probe) of the pancreatic head focusing on a normal-sized pancreatic duct.

ficult (Fig. 7). Notably, the morphologic characteristics of the lesion on EUS do not allow ready differentiation from pancreatic adenocarcinoma, or in rare cases, splenules in the pancreatic tail (which can mimic pancreatic NETs on CT). However, EUS-guided FNA can play an important role in differentiating these entities (Fig. 8) [16].

\section{Pancreatic adenocarcinoma}

Given its convenience, wide availability, and accuracy, there is little doubt that $\mathrm{CT}$ will remain the primary modality in the diagnosis and staging of pancreatic cancer for the foreseeable future. Nevertheless, EUS has been shown to be clearly superior to CT in its ability to detect pancreatic cancer, with sensitivities ranging from $96 \%$ to $98 \%$ [2, 12, 21, 22]. However, given the ability of CT to detect the vast majority of pancreatic cancers in a far less invasive fashion, the primary diagnostic utility of EUS is based upon: (1) the use of EUS in ambiguous cases where a mass is suspected, but cannot be absolutely confirmed on CT, and (2) the ability to perform EUSguided FNA. In addition, in rare cases, EUS has also been shown to upstage some pancreatic cancers by detecting vascular tumor spread, malignant ascites, or small liver metastases not visible on other imaging modalities, thus avoiding futile surgery [23, 24].

It is quite common for a pancreatic malignancy to be suspected on the basis of clinical presentation (i.e., jaundice, weight loss, etc.) and laboratory findings (elevated tumor markers), even though no discrete mass is identified on CT. In addition, it is also not uncommon to be confronted with subtle imaging findings on CT (i.e., focal enlargement of the pancreas, subtle hypodensity in 

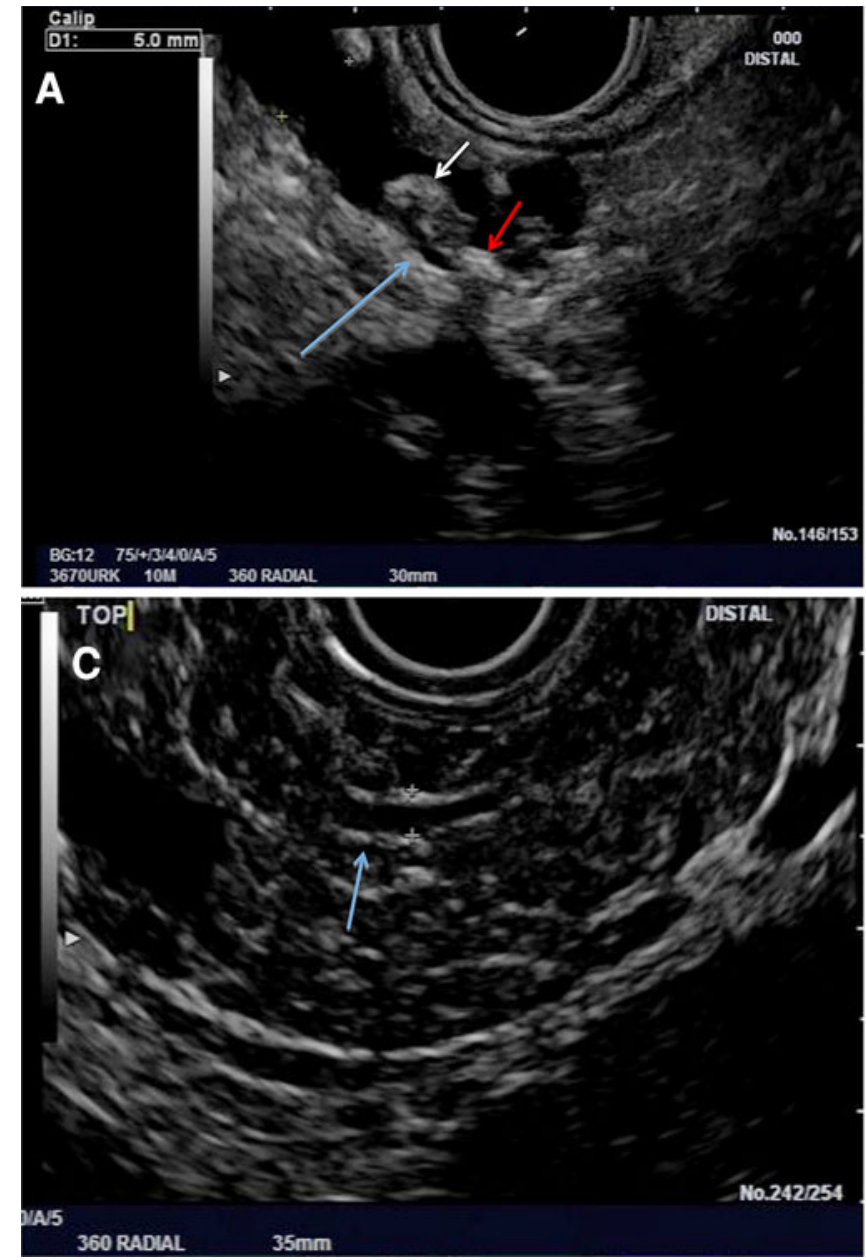

Fig. 6. Features of chronic pancreatitis: Endoscopic ultrasound image acquired with a radial probe $(\mathbf{A})$ demonstrates a dilated pancreatic duct (blue arrow) with internal stones (and posterior acoustic shadowing) (red arrow) and debris (white arrow). CT image (B) from the same patient as in A demon-

the pancreatic head), and to be unsure whether there is truly a mass present. Several studies have shown that EUS has a high negative predictive value, and that a negative EUS in such a setting can reliably exclude the presence of a malignancy [12]. In a study by Klapman et al. [25], 155 patients with suspected pancreatic cancer on the basis of clinical and laboratory findings were found to have a negative EUS, and none of these patients ultimately developed pancreatic cancer. In a study by Catanzaro et al. [26], 80 patients with suspected pancreatic cancer on the basis of CT or clinical/laboratory findings underwent a negative EUS, and none were found to ultimately develop pancreatic cancer. Similarly, in a group of patients with ambiguous pancreatic head enlargement on CT reported by Ho et al. [27], none of the patients with a negative EUS result had their diagnosis change with follow-up.

In most cases, the EUS appearance of pancreatic adenocarcinoma is akin to its appearance on

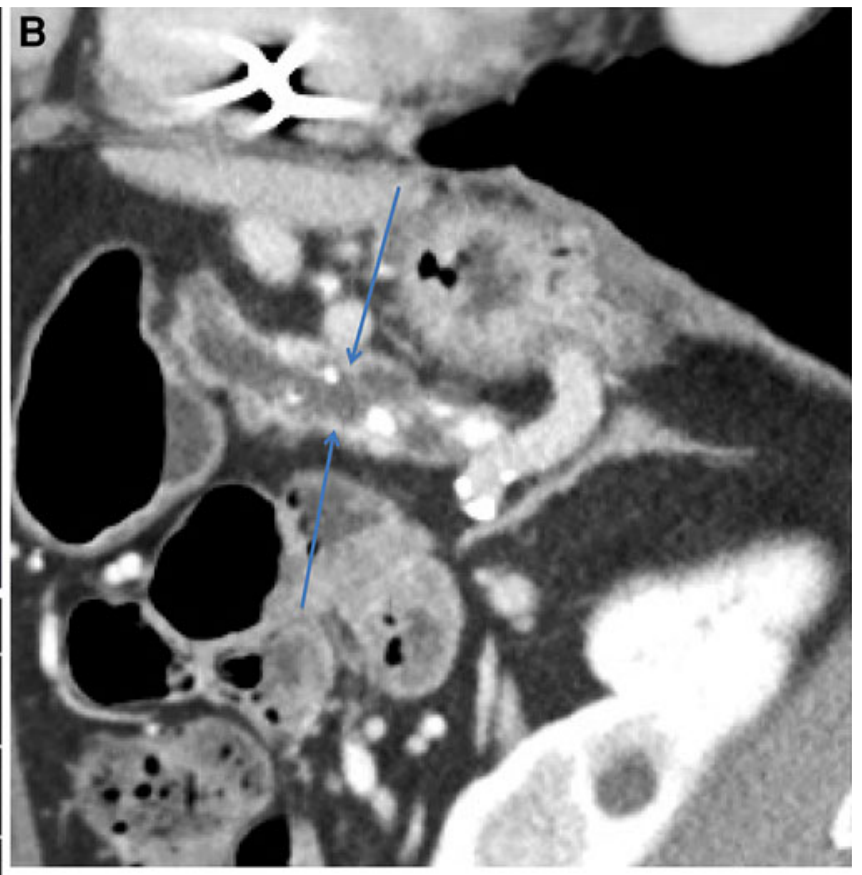

strates a markedly dilated pancreatic duct (arrows) with parenchymal and ductal calcifications. Endoscopic ultrasound image of a separate patient $(\mathbf{C})$ demonstrates a dilated main pancreatic duct (arrow) with hyperechoic walls (arrow), foci, and strands. The corresponding CT images in this patient were completely normal.

conventional transcutaneous ultrasound: A nonspecific, hypoechoic, poorly defined mass with upstream pancreatic ductal dilatation and abrupt pancreatic ductal cutoff (Fig. 8). Unfortunately, there are no specific EUS imaging features which can differentiate pancreatic cancer from other common mimics, including focal pancreatitis, NETs, lymphoma, metastases, and focal AIP (Fig. 9) [12]. Moreover, while there is some ambiguity in the literature, it is now felt that EUS is not as accurate in locoregional staging of pancreatic cancer or in the determination of resectability compared to CT [12].

Despite these weaknesses, one of the great strengths of EUS in pancreatic cancer is its ability to facilitate EUSguided FNA. In some centers, EUS-guided FNA has largely supplanted pancreatic biopsy performed using either conventional ultrasound or CT guidance, both of which are limited in some cases in their ability to visualize the tumor. Similarly, ERCP with cytologic brushings, which 

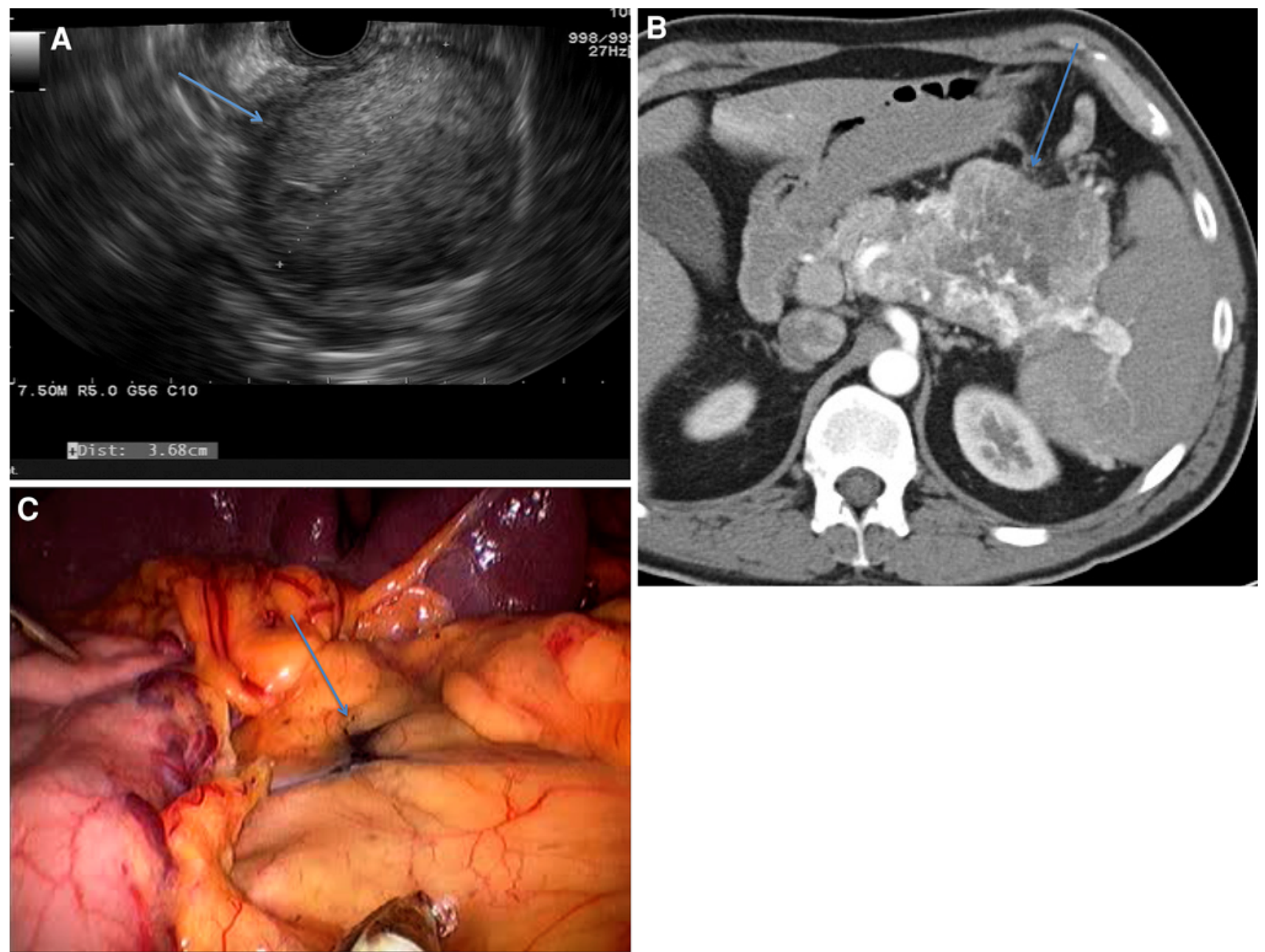

Fig. 7. Pancreatic neuroendocrine tumor: Endoscopic ultrasound image $(\mathbf{A})$ demonstrates a well-circumscribed mass (arrow) which is mildly hyperechoic. There are no specific sonographic features allowing this mass to be differentiated from a pancreatic adenocarcinoma or splenule. CT image from the

remains another option, is limited by its extremely poor sensitivity (as low as 30\% in some studies) [28].

EUS-FNA is performed using a linear echoendoscope, which allows the needle to be inserted into the pancreas under ultrasound guidance. EUS-FNA has proven to be quite safe, as a meta-analysis of over 10,000 patients found the overall rate of EUS-FNA-related morbidity to be $0.98 \%$ with a mortality of $0.02 \%$ [29]. EUS-FNA is very effective in confirming the presence of malignancy in solid pancreatic lesions, with an $85 \%$ sensitivity and $98 \%$ specificity [1]. If atypical or suspicious cytology (rather than definite malignancy) is used as the primary criterion, the sensitivity increases to $91 \%$ (but with a lower specificity of 94\%). In addition to its traditional role in biopsy, EUS is now increasingly being used to aid treatment of pancreatic adenocarcinoma through the placement of gold fiducial markers within the pancreatic mass, which are then used to target radiation treatment.

same patient (B) in the arterial phase demonstrates a large hypervascular mass (arrow) encompassing the pancreatic tail. Intraoperative photograph (C) (from a different patient) demonstrates an EUS placed "tattoo" marking the site of a neuroendocrine tumor, which was then used to guide resection.

It is now common for patients with pancreatic cancer deemed to be unresectable on the basis on CT to undergo EUS-guided FNA to facilitate further chemotherapy or radiation (by providing the oncologist with the tumor histology). Moreover, given the increasing availability of EUS, biopsies are now increasingly being obtained even in those patients with a tumor deemed to be resectable on $\mathrm{CT}$, in order to exclude mimics of pancreatic cancer, including lymphoma, focal pancreatitis, NETs, and metastases, all of which may have dramatically different treatments and prognoses (Fig. 10) [19].

\section{Pancreatic cystic lesions}

\section{Intraductal pancreatic mucinous neoplasm} (IPMN)

With improvements in CT and MRI scanner technology over the last decade, as well as improved understanding 


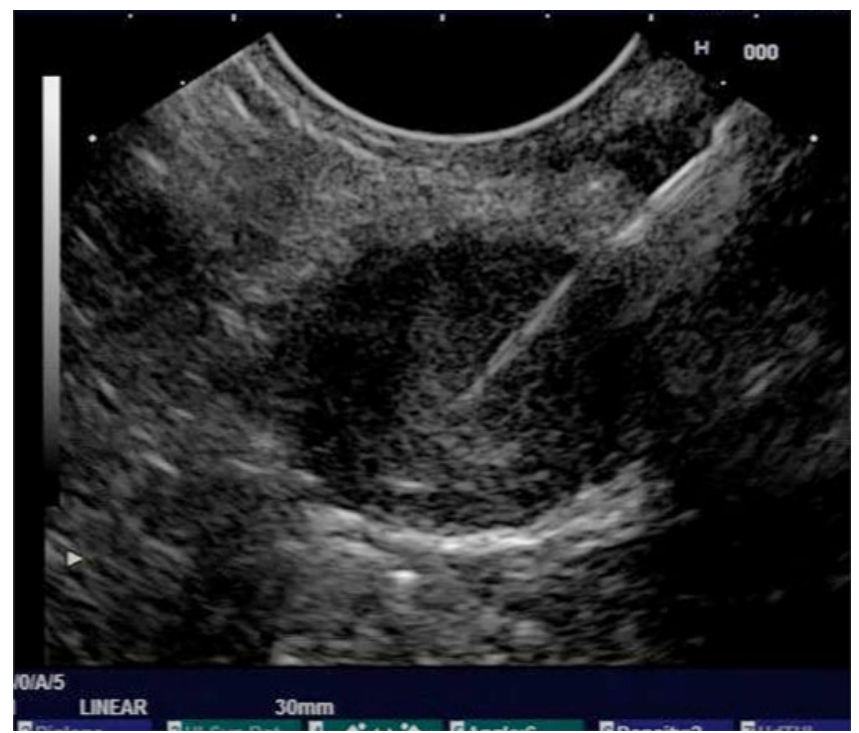

Fig. 8. Endoscopic ultrasound image demonstrates EUSguided FNA of a pathologically proven pancreatic neuroendocrine tumor. The biopsy was performed using a linear probe.

in the radiology community regarding their existence and significance, IPMNs are increasingly being identified on imaging studies. Incidental pancreatic cystic lesions are now identified in up to $2.6 \%$ of abdominal CTs [30] and up to $13.5 \%$ of MRIs, and the majority of these cystic lesions represent IPMNs [31].

IPMNs are mucin-producing lesions which can be divided into three types: (1) Side-branch IPMNs are

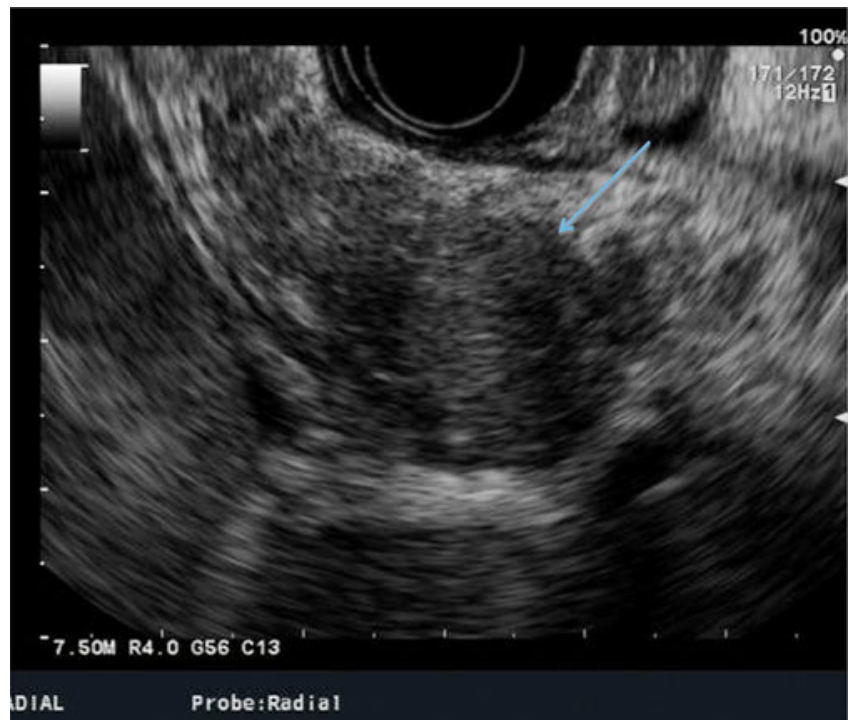

Fig. 10. Endoscopic ultrasound image acquired with a radial probe demonstrates a poorly marginated hypoechoic mass (arrow) which was originally thought to represent pancreatic adenocarcinoma, but which was ultimately found to represent focal pancreatitis. This distinction cannot be made based on the EUS morphology of the mass.

mucin-producing cystic neoplasms centered in a pancreatic duct side branch, which communicate with a normal main pancreatic duct, and have a risk of high grade dysplasia or invasive cancer of $24.4 \%$ and $16.6 \%$, respectively [32]. (2) Main duct IPMNs affect the main pancreatic duct, and demonstrate significantly higher risks of high grade dysplasia or invasive adenocarcinoma

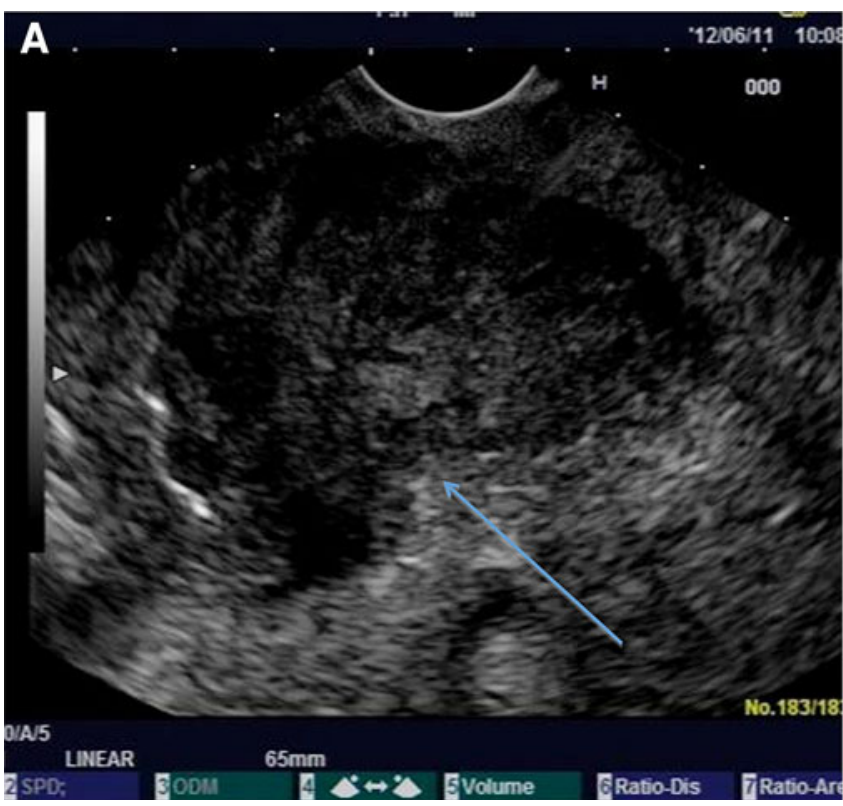

Fig. 9. Endoscopic ultrasound image (A) acquired using a linear probe demonstrates a hypoechoic mass (arrow) which was ultimately proven to represent a pancreatic adenocarci-

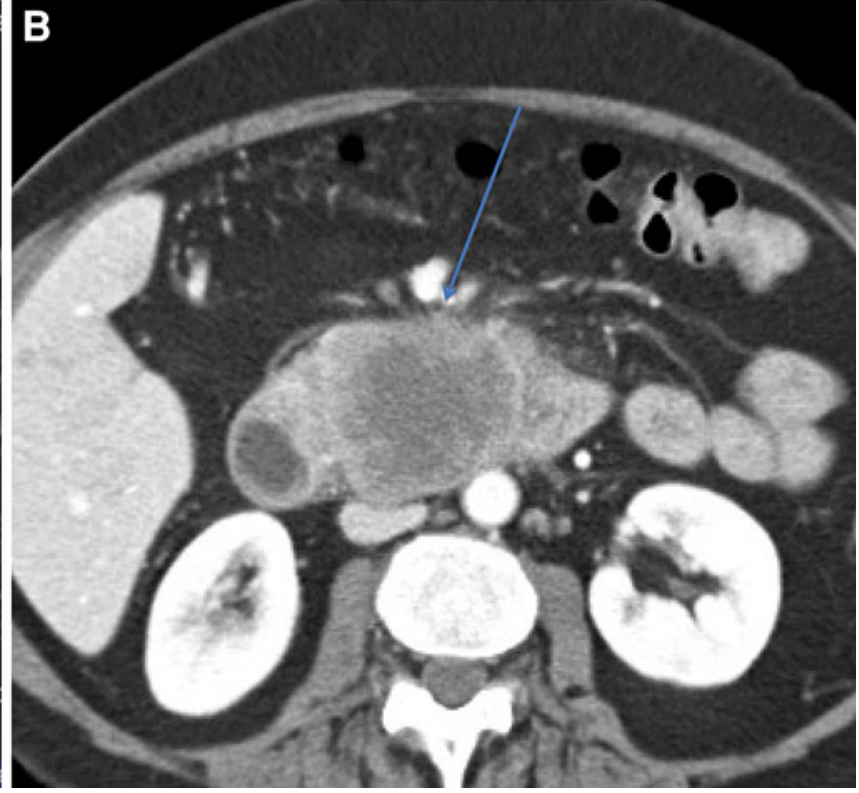

noma. CT image (B) from the same patient demonstrates a poorly marginated, hypodense mass (arrow) in the uncinate process. 

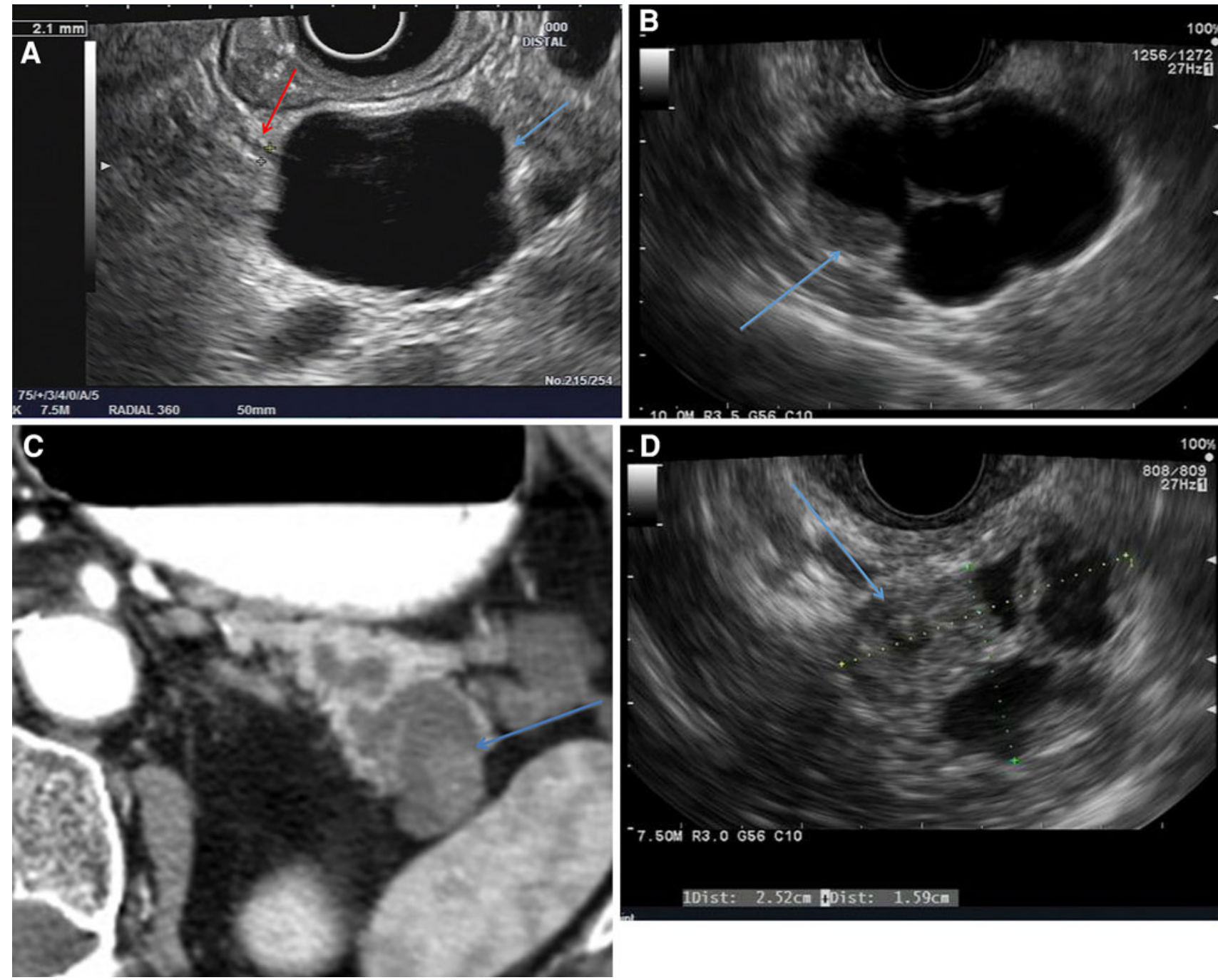

Fig. 11. Three different patients with side branch IPMNs. A A side-branch IPMN (blue arrow) with direct connection with the main pancreatic duct (red arrow). B A side-branch IPMN with discrete mural nodularity (arrow in B), a finding also visualized

(62.2\% and 57.6\%, respectively) [32]. (3) Mixed-type IPMNs combine elements of both main-duct and sidebranch type IPMNs and have a risk of malignant transformation similar to that of main duct IPMNs. All main-duct and mixed-type IPMNs should be resected, whereas the decision to resect a side-branch IPMN is more complex. In general, cysts demonstrating main pancreatic ductal dilatation $\geq 10 \mathrm{~mm}$, the presence of enhancing mural nodularity within a cyst or main pancreatic duct, and suspicious cytology, are all highly suspicious features which should warrant consideration for surgical resection (Fig. 10). The presence of pancreatitis, a cyst measuring $\geq 3 \mathrm{~cm}$, a thickened or enhancing cyst wall, a non-enhancing mural nodule, a main duct measuring 5-9 $\mathrm{mm}$, or an abrupt change in caliber of the pancreatic duct are considered concerning, but not definitively suspicious, features [32]. on the corresponding CT image (C) (arrow in $\mathbf{C}$-mural nodularity). D A solid mass (arrow) with internal anechoic cystic components. This mass was ultimately found to represent a pancreatic ductal adenocarcinoma arising from an IPMN.

Increasingly, EUS has become a vital component of the diagnostic algorithm for pancreatic cyst evaluation in the most specialized centers, both for diagnosis and risk stratification. From a morphologic standpoint, EUS is rarely absolutely diagnostic of a single entity, although identification of a connection between a cystic lesion and the main pancreatic duct is highly suggestive of a side branch IPMN (although this can also be present in pseudocysts) (Fig. 11) [33, 34]. These lesions can appear unilocular on EUS, or alternatively, multilocular with a "bunch of grapes" appearance. Dilation of the main pancreatic duct over $1 \mathrm{~cm}$ in diameter, particularly in cases where mucin is seen to be actively extruding from the ampulla, is a feature highly suggestive of a main duct IPMN (Fig. 12) [35]. In those cases where a cyst demonstrates a few concerning, but not absolutely suspicious, features, EUS can demonstrate morphologic 

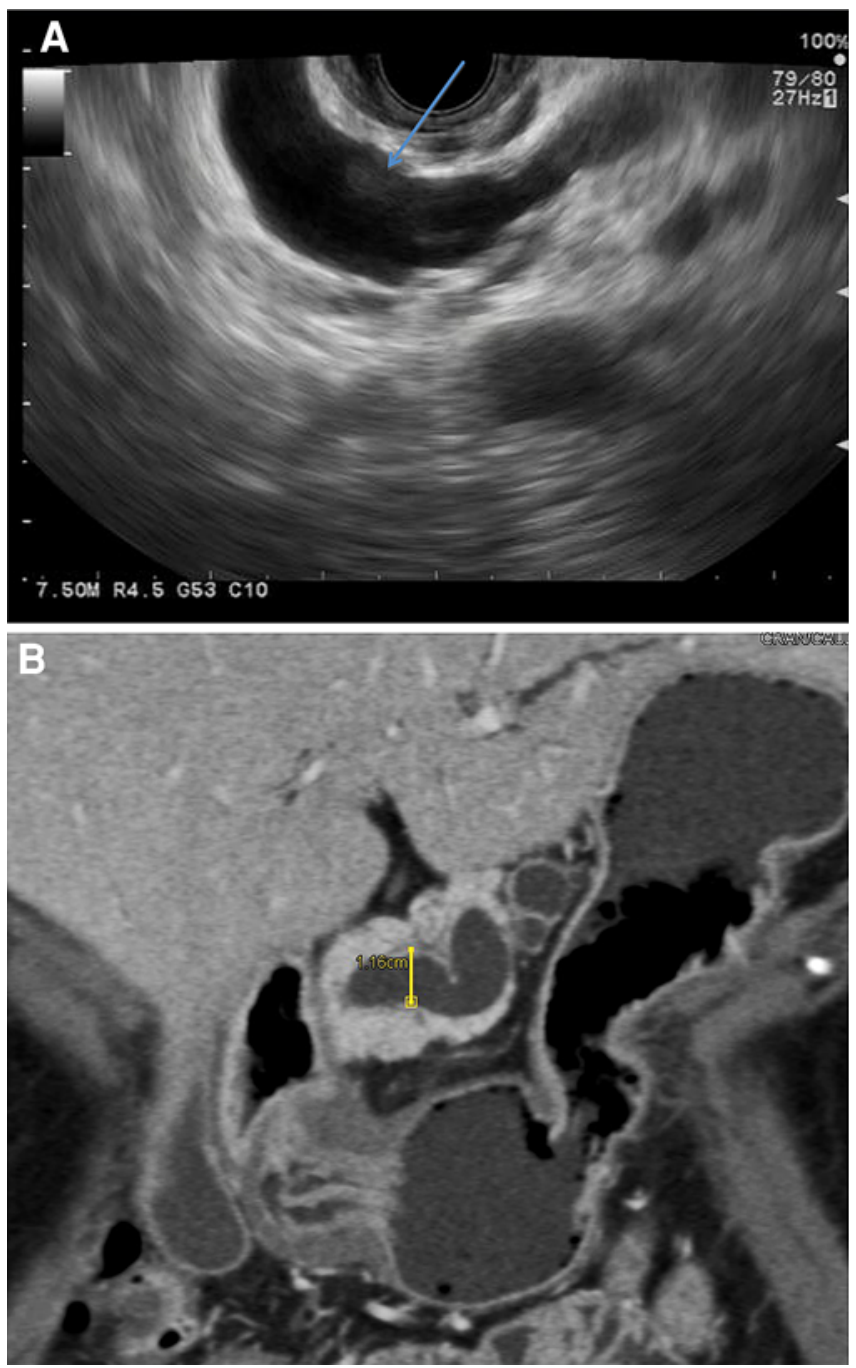

Fig. 12. Endoscopic ultrasound image $(\mathbf{A})$ demonstrates a markedly dilated main pancreatic duct with internal nodularity (arrow), an appearance highly suggestive of a malignant main duct IPMN. CT image from the same patient (B) demonstrates a markedly dilated main pancreatic duct, but the nodularity seen on EUS is not appreciated.

features suggestive of a malignant IPMN, including discrete mural nodularity within a dilated main pancreatic duct or cyst. While few studies have compared CT with EUS in terms of morphologic analysis, the superior spatial resolution of EUS undoubtedly can identify subtle mural nodules and solid components that are not visible on CT (Fig. 11).

While EUS may not often be able to differentiate side-branch IPMNs from other solitary cystic lesions on the basis of ultrasound morphology alone, EUS-guided FNA and cyst aspiration can play a major role in differentiating mucinous tumors from non-mucinous tumors. The presence of frank mucin (positive mucin stain) in the aspirated cyst contents, as well as elevated CEA levels, are both highly suggestive of a mucinous

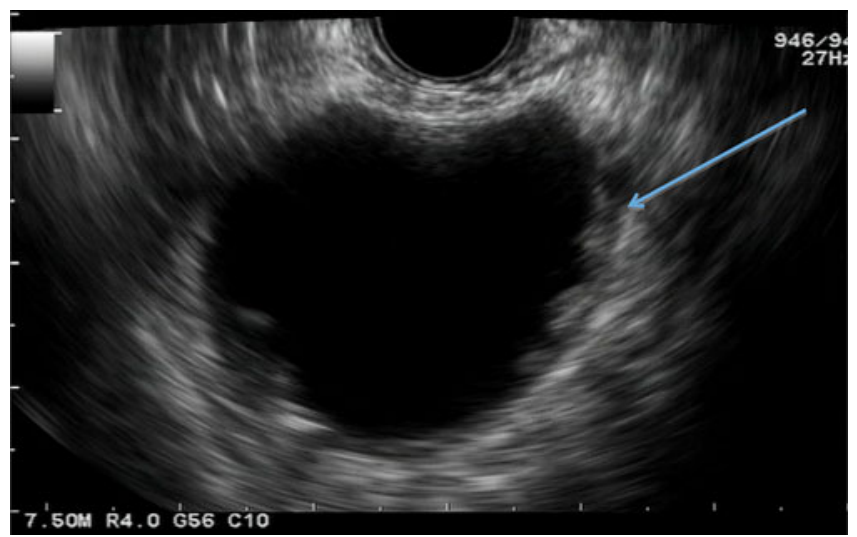

Fig. 13. Endoscopic ultrasound image demonstrates a cystic lesion (arrow) with minimal irregularity of the walls. This was found to represent an MCN, although the morphology does not allow this lesion to differentiated from other possible cystic lesions.

neoplasm (either IPMN or mucinous cystic neoplasm/ MCN [36]. In particular, a CEA level of $>192 \mathrm{ng} / \mathrm{mL}$ has been shown to have a sensitivity of $75 \%$ and a specificity of $84 \%$ in differentiating a mucinous from non-mucinous cyst $[2,37,38]$. Levels under $5 \mathrm{ng} / \mathrm{mL}$ suggest a benign pseudocyst or serous tumor [33]. It is important to note, however, that it is unclear whether CEA levels can be used to differentiate benign from malignant IPMNs [39]. Aspirated cyst fluid can also be sent for amylase levels to determine if a cyst has ductal communication, potentially differentiating an IPMN from a MCN $[34,39]$. In some cases, cytology can reveal evidence of atypia in the aspirated sample, a feature suggesting a greater risk of a malignant lesion: Notably, the presence of marked atypia on cytology is an indication for surgical resection [32]. Undoubtedly, the use of genetic markers from aspirated cyst fluid will play a major role in the decision to operate or observe cysts in the future.

Nevertheless, EUS-guided cyst aspiration is not without its limitations: Cysts must measure $1-2 \mathrm{~cm}$ in order to provide enough fluid to aspirate, as most laboratories require 1-2 cc of fluid to perform CEA analysis. In one study by de Jong et al., only $31 \%$ of samples had sufficient fluid for cytologic analysis, and only $49 \%$ had sufficient fluid for CEA analysis. The cytologic yield, in particular, can be poor, making any definite cytologic analysis by the pathologist difficult. In addition, roughly $10 \%$ of cysts cannot be punctured for aspiration as a result of small size or an inaccessible location [34].

\section{Mucinous cyst neoplasms (MCN)}

In most cases, EUS cannot reliably differentiate a MCN from other cystic lesions on the basis of morphology alone, although the presence of a thick wall, internal 

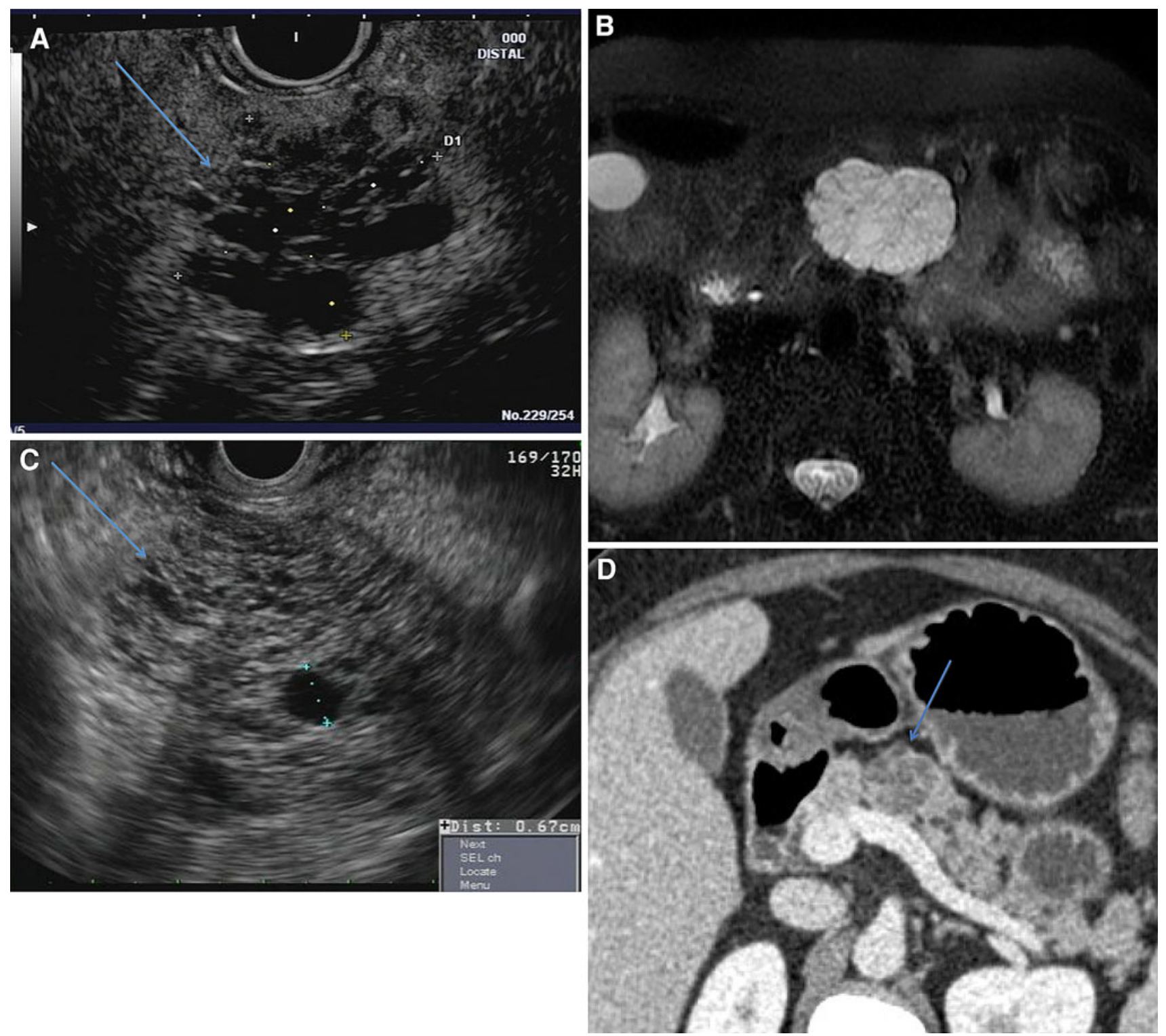

Fig. 14. Serous cystadenoma: A The classic "sponge" or "honeycomb" appearance of a serous cystadenoma (arrow). T2-weighted MRI image from the same patient (B) demonstrates a similar appearance, with a T2 hyperintense mass with multiple internal septations. C A serous cystadenoma (arrow)

mural nodularity, and solid components, are all features highly concerning for a malignancy (Fig. 13). These lesions do not usually demonstrate any connection with the adjacent pancreatic duct. In some cases, MCNs can appear as a solitary, round, unilocular cyst on EUS, although the presence of a few $(<6)$ macrocystic locules ("cyst within a cyst"), divided by septations, is the more typical appearance [35]. In rare cases, calcification can be appreciated in the cyst wall [36].

These lesions typically occur in women with a mean age of 45, and are almost always found in the body and tail of the pancreas [40]. MCNs are either premalignant

which appears nearly solid as a result of the large number of echogenic septations, although a few small cystic spaces are visible. Corresponding CT image from the same patient (D) demonstrates a hypodense mass (arrow) with a few internal microcystic components which are difficult to clearly identify.

or frankly malignant, with resection recommended in patients who are suitable surgical candidates. The diagnosis of MCN is often suspected on the basis of the cyst's location, the patient's age, the presence of macrocystic spaces within a cyst on EUS, and cyst aspiration results (elevated CEA levels, low amylase levels, mucin present on cytology).

\section{Serous cystadenoma}

Serous cystadenoma (SCA) is a rare tumor with a low risk of malignant transformation $(0-1.2 \%)$ [41-44]. The 

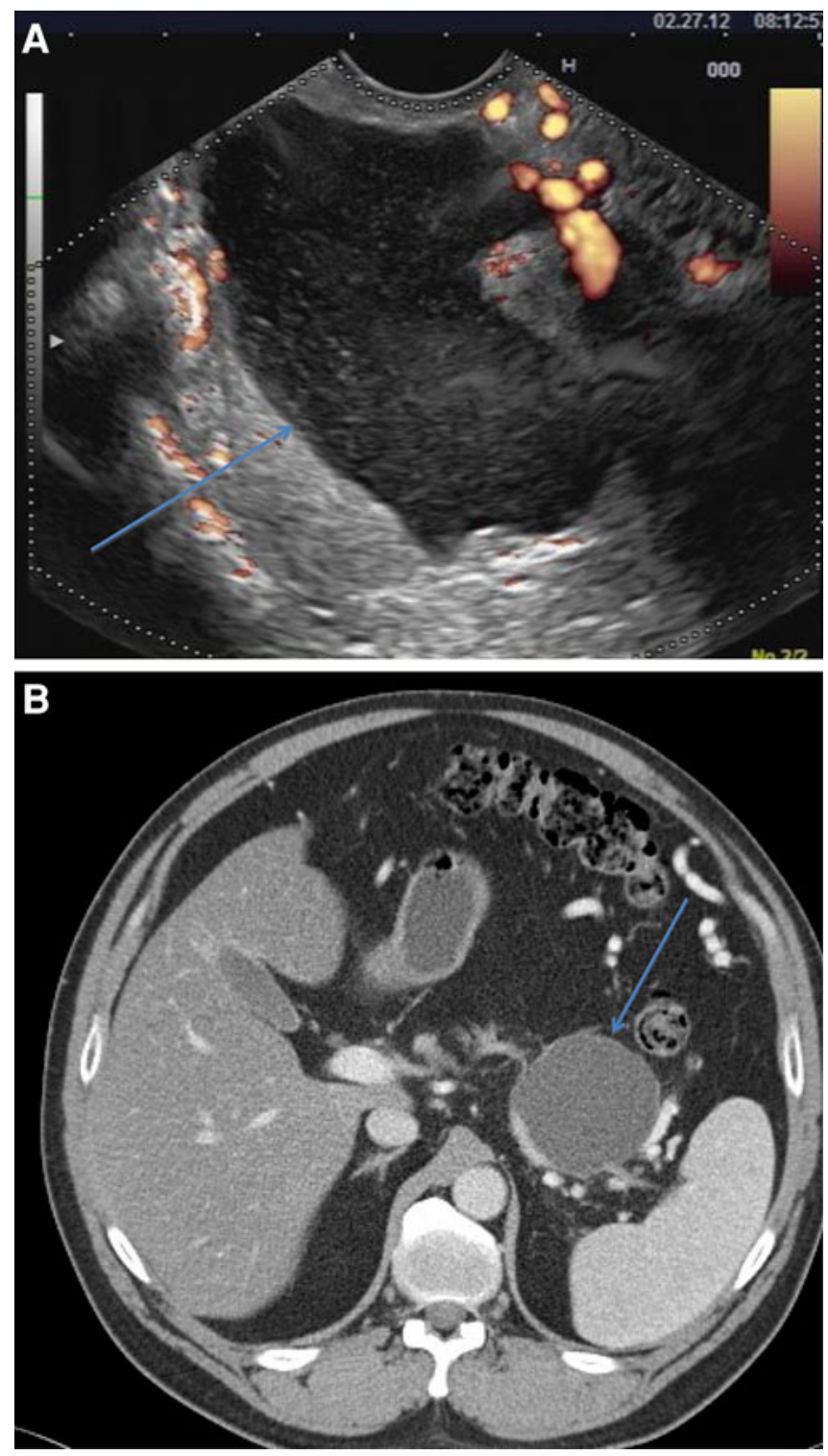

Fig. 15. Pseudocyst: Endoscopic ultrasound image (A) with power Doppler demonstrates a large avascular cystic lesion (arrow) with internal debris and complexity. CT image (B) from the same patient demonstrates a cystic mass (arrow) at the level of the pancreatic tail.

majority of these lesions occur in women, and tends to be equally distributed between the head, body, and tail. These tumors very rarely cause symptoms, even though they can be quite large when detected. The majority of SCAs demonstrate a microcystic morphology, and these microcystic lesions tend to be associated with the classic CT appearance (multiseptated small cysts with a central scar and calcification). In these classic cases, SCAs have a similar appearance on EUS, where they present with a "sponge" or "honeycomb" morphology (innumerable tiny cysts separated by septations, a central scar, and central calcification) (Fig. 14). Lesions with a typical appearance on CT require no further evaluation, and are simply followed with surveillance CT examinations.

However, 20\% of SCAs are macrocystic and oligocystic variants which have a very similar appearance on CT to a mucinous cystic neoplasm (MCN) or branch duct IPMN, and 3\% have a completely solid appearance which can mimic the appearance of a neuroendocrine tumour or adenocarcinoma (Fig. 14) [41]. This difficulty in correctly identifying the type of cystic pancreatic neoplasm is highlighted by a recent multicenter study of SCAs, which found that $31 \%$ were incorrectly identified as IPMN, NETs, MCN, ductal adenocarcinomas, or pseudocysts prior to resection [41]. EUS is particularly useful in the assessment of these solid or macrocystic/oligocystic SCAs, allowing cyst fluid sampling: Cytology typically shows cuboidal glycogen staining cells with no mucin staining. Cyst fluid CEA levels of $<5 \mathrm{ng} / \mathrm{mL}$ are associated with only a $50 \%$ sensitivity, but a $95 \%$ specificity, for SCA or pseudocysts. In addition, unlike pseudocysts, cyst aspirates typically demonstrate low amylase levels given the lack of ductal communication [45]. Although still not used in day-to-day practice, recent research has identified new molecular markers (GNAS/KRAS) which may further help differentiate SCA from IPMN. Notably, these two markers have been identified in the cyst fluid of $96 \%$ of patents with an IPMN, but none of the patients with SCA [46]. These results are very encouraging, but need validation in prospective studies before being incorporated into clinical practice.

\section{Pseudocyst}

Pseudocysts are incredibly common, accounting for a large proportion of all pancreatic cystic lesions, and are found in $20-40 \%$ of patients with chronic pancreatitis and $2-3 \%$ of patients with acute pancreatitis. There are no features on CT or MRI which can allow pseudocysts to be differentiated from a cystic pancreatic neoplasm, and the diagnosis is often inferred from a patient's history of prior bouts of pancreatitis [47]. However, it is not uncommon to identify cystic pancreatic neoplasms in patients with a history of prior pancreatitis, and one must always consider this possibility. While EUS typically does not provide any clear morphologic data differentiating a pseudocyst from a cystic neoplasm, the presence of a clear communication with the pancreatic duct can narrow the differential diagnosis to an IPMN or pseudocyst (Fig. 15). Moreover, cyst aspiration can also be a valuable tool in these cases, as pseudocysts often contain neutrophils, macrophages, plasma cells, and other inflammatory debris in their aspirated fluid. In cases where a pseudocyst is suspected, cyst fluid amylase should be analyzed, as a low cyst fluid amylase of $<250 \mathrm{U} / \mathrm{L}$ virtually excludes the diagnosis of a pseudocyst [45]. In patients who are symptomatic, internal drainage of pancreatic pseudocysts through a transgastric or transduodenal route can also be performed using EUS [48]. 


\section{Conclusion}

Over the last 25 years, EUS has gradually made significant inroads into pancreatic imaging, and now plays a significant diagnostic role in the evaluation of both solid and cystic pancreatic lesions. EUS has shown great utility not only in its ability to evaluate the morphologic features of lesions, but also in its ability to readily biopsy lesions in and around the pancreas which are not always readily amenable to biopsy under $\mathrm{CT}$ or conventional ultrasound guidance. It is important for radiologists to be aware of current EUS capabilities, as EUS may often be the recommended follow-up examination once a lesion is identified on CT or MRI. Moreover, as the diagnostic algorithm for a number of different pancreatic disorders grows more sophisticated, CT, MRI, and EUS must be looked upon as complementary tools in the evaluation of pancreatic disease.

\section{References}

1. Hewitt MJ, McPhail MJ, Possamai L, et al. (2012) EUS-guided FNA for diagnosis of solid pancreatic neoplasms: a meta-analysis. Gastrointest Endosc 75(2):319-331

2. Chaya CT, Bhutani MS (2007) Ultrasonography of the pancreas. 6. Endoscopic imaging. Abdom Imaging 32(2):191-199

3. Reddy Y, Willery RP (2009) Endoscopic ultrasound: what is it and when should it be used? Clin Med 9(6):539-543

4. Palazzo L, Lennon AM, Penman ID (2011) Endosonography. In: Canard JM, Letard J-C, Palazzo L, Penman ID, Lennon AM (eds) Gastrointestinal endoscopy in practice. Amsterdam: Elsevier, pp 274-370

5. Vandervoort J, Soetikno RM, Tham TC, et al. (2002) Risk factors for complications after performance of ERCP. Gastrointest Endosc 56(5):652-656

6. Garcia-Cano Lizcano J, Gonzalez Martin JA, Morillas Arino J, Perez Sola A (2004) Complications of endoscopic retrograde cholangiopancreatography. A study in a small ERCP unit. Rev Esp Enferm Dig 96(3):163-173

7. Catalano MF (2007) Diagnosing early-stage chronic pancreatitis: is endoscopic ultrasound a reliable modality? J Gastroenterol 42(Suppl 17):78-84

8. Stevens T (2010) Endoscopic ultrasound for the diagnosis of chronic pancreatitis. World J Gastroenterol 16(23):2841

9. Wallace MB, Hawes RH, Durkalski V, et al. (2001) The reliability of EUS for the diagnosis of chronic pancreatitis: interobserver agreement among experienced endosonographers. Gastrointest Endosc 53(3):294-299

10. Chong AK, Hawes RH, Hoffman BJ, et al. (2007) Diagnostic performance of EUS for chronic pancreatitis: a comparison with histopathology. Gastrointest Endosc 65(6):808-814

11. Kahl S, Glasbrenner B, Leodolter A, et al. (2002) EUS in the diagnosis of early chronic pancreatitis: a prospective follow-up study. Gastrointest Endosc 55(4):507-511

12. Papanikolaou IS, Adler A, Neumann U, Neuhaus $P$, Rosch T (2009) Endoscopic ultrasound in pancreatic disease - its influence on surgical decision-making. An update 2008. Pancreatology 9(12):55-65

13. Hoki N, Mizuno N, Sawaki A, et al. (2009) Diagnosis of autoimmune pancreatitis using endoscopic ultrasonography. J Gastroenterol 44(2):154-159

14. Farrell JJ, Garber J, Sahani D, Brugge WR (2004) EUS findings in patients with autoimmune pancreatitis. Gastrointest Endosc 60(6):927-936

15. Chari ST, Takahashi N, Levy MJ, et al. (2009) A diagnostic strategy to distinguish autoimmune pancreatitis from pancreatic cancer. Clin Gastroenterol Hepatol 7(10):1097-1103

16. Patel KK, Kim M (2008) Neuroendocrine tumors of the pancreas: endoscopic diagnosis. Curr Opin Gastroenterol 24:638-642
17. Rosch T, Lightdale CJ, Botet JF, et al. (1992) Localization of pancreatic endocrine tumors by endoscopic ultrasonography. $\mathrm{N}$ Engl J Med 326(26):1721-1726

18. Zimmer T, Scherubi H, Faiss S, et al. (2000) Endoscopic ultrasound of neuroendocrine tumors. Digestion 62:45-50

19. Varadarajulu S, Eloubeidi MA (2010) The role of endoscopic ultrasonography in the evaluation of pancreatico-biliary cancer. Surg Clin North Am 90(2):251-263

20. Lennon AM, Newman N, Makary MA, et al. (2010) EUS-guided tattooing before laparoscopic distal pancreatic resection (with video). Gastrointest Endosc 72(5):1089-1094

21. Lennon AM, Penman ID (2007) Endoscopic ultrasound in cancer staging. Br Med Bull 84:81-98

22. Nichols MT, Russ PD, Chen YK (2006) Pancreatic imaging: current and emerging technologies. Pancreas 33:211-220

23. Levy MJ, Gleeson FC, Zhang L (2009) Endoscopic ultrasound fineneedle aspiration detection of extravascular migratory metastasis from a remotely located pancreatic cancer. Clin Gastroenterol Hepatol 7(2):246-248

24. DeWitt J, Yu M, Al-Haddad MA, et al. (2010) Survival in patients with pancreatic cancer after the diagnosis of malignant ascites or liver metastases by EUS-FNA. Gastrointest Endosc 71(2):260-265

25. Klapman JB, Chang KJ, Lee JG, Nguyen P (2005) Negative predictive value of endoscopic ultrasound in a large series of patients with a clinical suspicion of pancreatic cancer. Am J Gastroenterol 100(12):2658-2661

26. Catanzaro A, Richardson S, Veloso H, et al. (2003) Long-term follow-up of patients with clinically indeterminate suspicion of pancreatic cancer and normal EUS. Gastrointest Endosc 58:836840

27. Ho S, Bonasera RJ, Pollack BJ, et al. (2006) A single-center experience of endoscopic ultrasound for enlarged pancreas on computed tomography. Clin Gastroenterol Hepatol 4(1):98-103

28. Chang KJ (2006) State of the art lecture: endoscopic ultrasound (EUS) and FNA in pancreatico-biliary tumors. Endoscopy 38:S56S60

29. Wang KX, Ben QW, Jin ZD, et al. (2011) Assessment of morbidity and mortality associated with EUS-guided FNA: a systematic review. Gastrointest Endosc 73(2):283-290

30. Laffan TA, Horton KM, Klein AP, et al. (2008) Prevalence of unsuspected pancreatic cysts on MDCT. AJR Am J Roentgenol 191(3):802-807

31. Lee KS, Sekhar A, Rofsky NM, Pedrosa I (2010) Prevalence of incidental pancreatic cysts in the adult population on MR imaging. Am J Gastroenterol 105(9):2079-2084

32. Tanaka M, Fernandez-Del Castillo C, Adsay V, et al. (2012) International consensus guidelines 2012 for the management of IPMN and MCN of the pancreas. Pancreatology 12(3):183-197

33. Cocieru A, Brandwein S, Saldinger PF (2011) The role of endoscopic ultrasound and cyst fluid analysis in the initial evaluation and follow-up of incidental pancreatic cystic lesions. HPB 13(7):459-462

34. Hawes RH, Clancy J, Hasan MK (2012) Endoscopic ultrasoundguided fine needle aspiration in cystic pancreatic lesions. Clin Endosc 45(2):128-131

35. Barresi L, Tarantino I, Granata A, Curcio G, Traina M (2012) Pancreatic cystic lesions: how endoscopic ultrasound morphology and endoscopic ultrasound fine needle aspiration help unlock the diagnostic puzzle. World J Gastrointest Endosc 4(6):247-259

36. Petrone MC, Arcidiacono PG (2008) Role of endosocopic ultrasound in the diagnosis of cystic tumours of the pancreas. Dig Liver Dis $40(11): 847-853$

37. Scheiman JM (2008) Management of cystic lesions of the pancreas. J Gastrointest Surg 12(3):405-407

38. Visser BC, Muthusamy VR, Yeh BM, Coakley FV, Way LW (2008) Diagnostic evaluation of cystic pancreatic lesions. HPB 10(1):63-69

39. Larghi A, Petrone MC, Galasso D, Arcidiacono PG (2010) Endoscopic ultrasound in the evaluation of pancreaticobiliary disorders. Dig Liver Dis 42(1):6-15

40. Yamao K, Yanagisawa A, Takahashi K, et al. (2011) Clinicopathological features and prognosis of mucinous cystic neoplasm with ovarian-type stroma: a multi-institutional study of the Japan pancreas society. Pancreas 40(1):67-71 
41. Kimura W, Moriya T, Hanada K, et al. (2012) Multicenter study of serous cystic neoplasm of the Japan pancreas society. Pancreas 41(3):380-387

42. Galanis C, Zamani A, Cameron JL, et al. (2007) Resected serous cystic neoplasms of the pancreas: a review of 158 patients with recommendations for treatment. J Gastrointest Surg 11(7):820-826

43. Tseng JF, Warshaw AL, Sahani DV, et al. (2005) Serous cystadenoma of the pancreas: tumor growth rates and recommendations for treatment. Ann Surg 242(3):413-419 (discussion 9-21)

44. Khashab MA, Shin EJ, Amateau S, et al. (2011) Tumor size and location correlate with behavior of pancreatic serous cystic neoplasms. Am J Gastroenterol 106(8):1521-1526
45. van der Waaij LA, van Dullemen HM, Porte RJ (2005) Cyst fluid analysis in the differential diagnosis of pancreatic cystic lesions: a pooled analysis. Gastrointest Endosc 62(3):383-389

46. Wu J, Matthaei H, Maitra A, et al. (2011) Recurrent GNAS mutations define an unexpected pathway for pancreatic cyst development. Sci Transl Med 3(92):92ra66

47. Spence RA, Dasari B, Love M, Kelly B, Taylor M (2011) Overview of the investigation and management of cystic neoplasms of the pancreas. Dig Surg 28(5-6):386-397

48. Khashab MA, Lennon AM, Singh VK, et al. (2010) EUS-guided pseudocyst drainage as a one-step procedure using a novel multiple wire insertion technique. Endoscopy 42(Suppl 2):E292-E293 